\title{
Thermodynamics-A Science of the 19th Century?
}

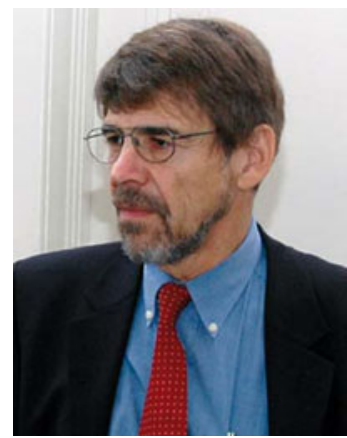

Chemical Thermodynamics is traditionally one of the subjects chemistry students learn in courses on Physical Chemistry. Students in Materials Science or Metallurgy obtain frequently a short introduction into the basics of thermodynamics without much background information. On the other hand, the use of phase diagrams has been taught for many decades in a more or less empirical way without much reference to the thermodynamic background. Many students memorized the Laws of Thermodynamics without asking for the reasons behind them, or they constructed more or less complicated phase diagrams without thinking about the background of the phase rule. They all passed their exams but many of them hated this subject and didn't see any reasonable use for it.

When I was an undergraduate chemistry student in the nineteen-sixties the introductory course on Physical Chemistry started with basic chemical thermodynamics and the first test in the corresponding laboratory course was again about thermodynamics. As many of my colleagues I passed the exam and finished the lab course with a reasonable grade but had not really any idea why the knowledge of thermodynamic relationships could be important.

Then, when I started my doctoral thesis (with Kurt Komarek at the University of Vienna) I was asked to measure tellurium vapor pressures in the binary iron-tellurium system, to calculate partial thermodynamic properties of tellurium from the results and to apply statistical-thermodynamic model calculations to learn more about the defect chemistry in one of the nonstoichiometric intermetallic phases. And I was also asked to clarify the corresponding binary phase diagram. Suddenly I started to understand that thermodynamics is more than just laws and equations, and that the Gibbs energy really tells us something about the stability — or at least relative stability — of various materials and that, maybe, statistical thermodynamics might be quite interesting after all.

As a Postdoc I had the chance to work for two years with Austin Chang in the US, and again we used statistical thermodynamics to evaluate our experimental results and learn more about defect mechanisms in non-stoichiometric intermetallic compounds. And I began to appreciate the power of statistical thermodynamics for understanding why point defects are present in solids and why particular types of point defects prevail in certain materials. To some extent I started to like thermodynamics.

This was also the time when Larry Kaufman had published his famous book (together with Harold Bernstein) "Computer Calculation of Phase Diagrams" (Academic Press, 1970) where he emphasized the intricate interconnection between thermodynamics and phase equilibria in materials and showed ways how to calculate phase equilibria from known thermodynamic properties, with a possibility to extrapolate these to other temperature and composition ranges. This was the birth of the CALPHAD technique. The CALPHAD method has been used and further expanded since then, and today it is a powerful standard method to obtain optimized and consistent phase diagrams of binary, ternary, and higher order materials systems. Unfortunately, many scientists have been using this method and the available software packages as a "black box" to calculate phase equilibria without proper consideration (or proper knowledge?) of the necessary scientific background, producing "assessed phase diagrams" which are useless because they are-at the best—a good guess only.

Later in my scientific career I had the chance to meet eminent scientists like Philipp Gross and, especially, Oswald Kubaschewski who showed how thermodynamic data can be used to design technological processes and to estimate their efficiency. This helped to finally convince me that thermodynamics is not "Science of the 19th Century" (as one of my chemistry colleagues claims) but that it is a useful and necessary basis for a huge number of possible applications.

Thermodynamics has also been the topic of my cooperation with scientists from various parts of the world, and it is alarming that many laboratories have closed down their corresponding facilities. Only few places are left worldwide with the competence to experimentally measure thermodynamic properties of materials at elevated temperatures. But the need for reliable data is still there. One example may be their importance in nuclear technology for an understanding of the stability of virtually all structural and functional materials. How could we tell if a certain material is compatible with a particular type of nuclear fuel and the fission products generated if we don't know their thermodynamic properties? Or how could we tell what type of material can be used to carry liquid sodium or other liquid alloys as coolants, or how clean the materials must be, in order not to react with each other with catastrophic failures as a consequence? (And that goes far beyond nuclear technology.) Many results of basic research must be carried together, like pieces of a complicated puzzle, in order to provide the necessary 
thermodynamic information. And only with this basic knowledge in their back, engineers can start to design the corresponding facilities.

Thus it is to be hoped that the remaining "thermodynamicists" will be able to pass on their expertise to the young scientists and that some tradition will carry on into the future. I am convinced that future generations will desperately need this knowledge and that it will still take decades until theoreticians may be able to provide all the necessary data without any experiments.

Herbert Ipser

Associate Editor

Journal of Phase Equilibria and Diffusion 